\title{
Strain Rate Induced Crystallization in Bulk Metallic Glass-Forming Liquid
}

\author{
Boonrat Lohwongwatana \\ Keck Laboratory of Engineering Materials, California Institute of Technology, Pasadena, California 91125, USA \\ Jan Schroers* \\ Keck Laboratory of Engineering Materials, California Institute of Technology, Pasadena, California 91125, USA, \\ and Liquidmetal Technologies, Lake Forest, California 92630, USA \\ William L. Johnson \\ Keck Laboratory of Engineering Materials, California Institute of Technology, Pasadena, California 91125, USA
}

(Received 30 June 2005; published 22 February 2006)

\begin{abstract}
We report on the solidification of $\mathrm{Au}_{49} \mathrm{Ag}_{5.5} \mathrm{Pd}_{2.3} \mathrm{Cu}_{26.9} \mathrm{Si}_{16.3}$ bulk metallic glass under various strain rates. Using a copper mold casting technique with a low strain rate during solidification, this alloy is capable of forming glassy rods of at least $5 \mathrm{~mm}$ in diameter. Surprisingly, when the liquid alloy is splat cooled at much higher cooling rates and large strain rates, the solidified alloy is no longer fully amorphous. Our finding suggests that the large strain rate during splat cooling induces crystallization. The pronounced difference in crystallization behavior cannot be explained by the previously observed strain rate effect on viscosity alone. A strain rate induced phase separation process is suggested as one of the explanations for this crystallization behavior. The strain-rate-dependent critical cooling rate must be considered in order to assess the intrinsic glass forming ability of metallic liquid.
\end{abstract}

DOI: 10.1103/PhysRevLett.96.075503

In the 1950s, Turnbull predicted that glass formation in metals is possible if heterogeneous nucleation could be suppressed [1]. Shortly after, Duwez and co-workers reported the synthesis of the first metallic glass [2] by rapidly cooling an Au-Si alloy at a rate of approximately $10^{6} \mathrm{~K} / \mathrm{s}$. In the 1970s, ribbons, splats, powder, droplets, wires, and thin films were the typical forms of early metallic glasses obtained by rapid quenching [3,4]. Pd-Cu-Si alloy system was the first exception-Chen's discovery of this alloy system provided the first evidence that metallic glass could be cast in bulk form [5].

During the past few decades, bulk metallic glasses have been discovered in a wide range of alloys [6-11]. In some alloys, a critical cooling rate to avoid crystallization as low as $0.005 \mathrm{~K} / \mathrm{s}$ [12] and critical casting thickness of up to $7 \mathrm{~cm}$ were reported [13] when heterogeneous nucleation was reduced.

With the exception of some exotic bulk metallic glass (BMG) synthesis methods [14-17], most amorphous alloys have been created by cooling the liquid from above its liquidus temperature. In this case, the intrinsic glassforming ability (GFA) and critical casting thickness, $d_{\max }$, were understood to be completely characterized by the critical cooling rate, $R_{c}$ (see, e.g., [18].)

This Letter presents solidification studies on gold bulk metallic glass-forming alloy $\mathrm{Au}_{49} \mathrm{Ag}_{5.5} \mathrm{Pd}_{2.3} \mathrm{Cu}_{26.9} \mathrm{Si}_{16.3}$, which could be cast into fully amorphous rods of at least $5 \mathrm{~mm}$ in diameter using a conventional copper mold injection method. When the liquid alloy was splat cooled, where it was exposed to both high cooling rate and high strain rate, it crystallized during solidification. This suggests that the high strain rate induces crystallization. This effect
PACS numbers: 61.43.Dq, 64.70.Dv, 64.75.+g, 82.60.Lf

becomes less pronounced with increasing processing temperature.

Ingots of $\mathrm{Au}_{49} \mathrm{Ag}_{5.5} \mathrm{Pd}_{2.3} \mathrm{Cu}_{26.9} \mathrm{Si}_{16.3}$ alloy were prepared by arc-melting the elements (purity: $\mathrm{Au}, 99.95 \% ; \mathrm{Cu}$, 99.9\%; Ag, 99.5\%; Pd, 99.95\%; Si, 99.95\%) in a titaniumgettered, argon-filled atmosphere. Fully amorphous $5 \mathrm{~mm}$ thick rectangular strips were produced using a copper mold injection casting technique. The bulk glassy strips were cut into small cubic pieces of approximately $1 \mathrm{~mm}^{3}$ in volume. Each sample was heated in a levitation coil to various temperatures ranging from 900 to $1500 \mathrm{~K}$ under $10^{-6} \mathrm{mbar}$ vacuum, and then splatted into thin foils of various thicknesses. Thermal analysis was performed in a Netzsch DSC 404c differential scanning calorimeter (DSC) and Perkin Elmer DSC7. X-ray diffraction analysis (XRD) was carried out on an Inel XRG 3000 using $\mathrm{Cu} \mathrm{K} \alpha$ radiation.

Figure 1 compares the XRD spectrum of (a) a $50 \mu \mathrm{m}$ splat-cooled sample processed at $\sim 900 \mathrm{~K}$, (b) a $300 \mu \mathrm{m}$ splat-cooled sample processed at $\sim 900 \mathrm{~K}$, and (c) a $5 \mathrm{~mm}$ copper mold cast alloy. The spectrum of the $50 \mu \mathrm{m}$ splatcooled sample consists mainly of narrow peaks, indicating a substantial amount of crystallinity. The spectrum of the $300 \mu \mathrm{m}$ splat-cooled spectrum indicates a detectable amount of crystallinity embedded in the amorphous matrix. The spectrum of the $5 \mathrm{~mm}$ cast sample was taken from the cross section and exhibited two broad diffraction peaks, a typical signature of a fully amorphous sample.

The DSC thermograms measured while continuously heating at $0.33 \mathrm{~K} / \mathrm{s}$ are shown in Fig. 2. For the copper mold cast sample, thermogram (c) shows a glass transition temperature, $T_{g}$, of $403 \mathrm{~K}$ and a crystallization temperature, $T_{x}$, of $461 \mathrm{~K}$. The ratio of the heat of crystallization to 


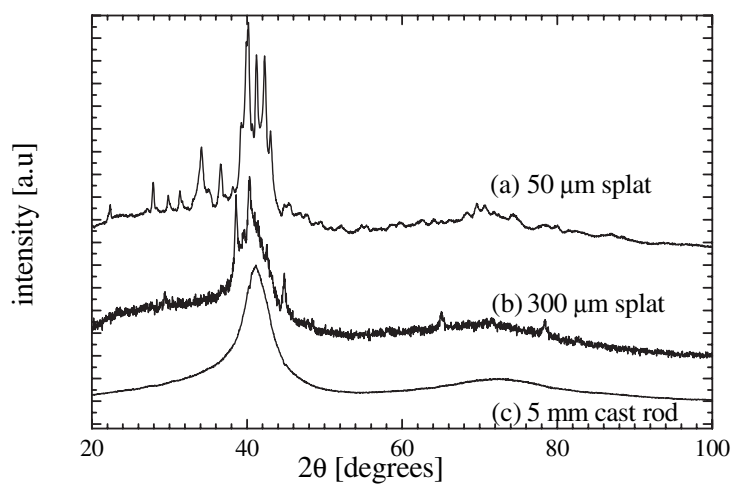

FIG. 1. X-ray diffraction spectrum for (a) $50 \mu \mathrm{m}$ splat-cooled sample, (b) $300 \mu \mathrm{m}$ splat-cooled sample, and (c) $5 \mathrm{~mm}$ cast rod. All samples were processed at $\sim 900 \mathrm{~K}$.

the heat of fusion is 0.75 , confirming that the material was entirely amorphous [11]. Thermogram (a) of the $50 \mu \mathrm{m}$ splat-cooled sample does not show an obvious heat release during heating, indicating the crystalline nature of the splat. Thermogram (b) reveals the heat of crystallization to heat of fusion ratio of $\sim 0.4$, confirming that the $300 \mu \mathrm{m}$ splat is partially crystalline. The solidification temperatures, $T_{m}$, for all three samples are in agreement with one another implying that the nominal composition of the splat has not been altered.

To further investigate our findings, the same alloy was subjected to different strain rates and processing temperatures. The results are summarized in Table I. The splat foils were obtained from using the aforementioned splat quencher. The pistons speed was varied to obtain different foil thicknesses. The cast foil of $30 \mu \mathrm{m}$ was obtained by water quenching in thin walled silicon molds. The millimeter level cast strips were obtained using injection copper mold casting technique. It was found that the crystallization was bypassed if the samples were subjected to low strain rates. The processing temperature does not have an effect on the suppression of crystallization if the strain

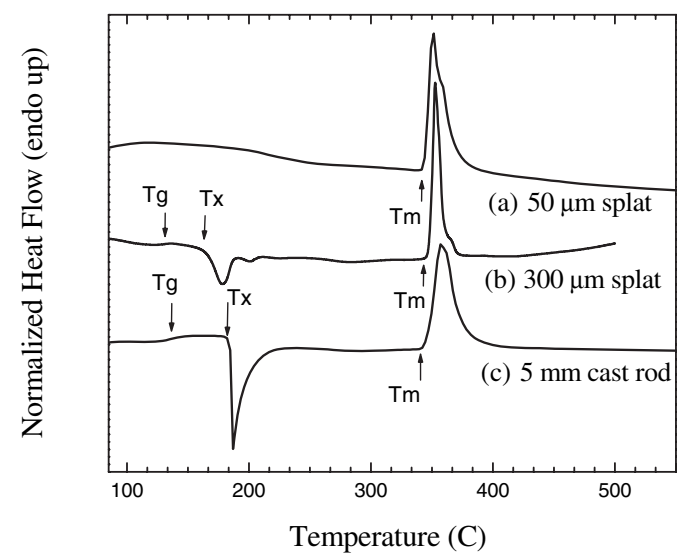

FIG. 2. Differential scanning calorimetry thermograms determined at $0.33 \mathrm{~K} / \mathrm{s}$ heating rate for (a) $50 \mu \mathrm{m}$ splat-cooled sample, (b) $300 \mu \mathrm{m}$ splat-cooled sample, and (c) $5 \mathrm{~mm}$ cast rod. All samples were processed at $\sim 900 \mathrm{~K}$. rates are low. However, when the alloy is exposed to a high strain rate (4000-10000 s ${ }^{-1}$ ) [20], crystallization occurs even though the cooling rates are in the order of $10^{5}-10^{6} \mathrm{~K} / \mathrm{s}$. This crystallization effect was found to be influenced by the processing temperature. With increasing processing temperature, the crystalline volume fraction decreases and, at about $1500 \mathrm{~K}$, the sample becomes mainly amorphous.

To date, no strain rate effects on the crystallization in metallic liquid have been reported. However, for several metallic glasses, it has been found that the strain rate has an effect on the viscosity [21-24]. These non-Newtonian effects result in a decrease of the viscosity with increasing strain rate. The viscosity has a strong influence on the crystallization kinetics. According to classical nucleation theory, the time for crystallization, $t_{x}$, is proportional to [25]

$$
t_{x} \propto\left(\frac{1}{I_{S S} u^{3}}\right)^{1 / 4},
$$

where $I_{s s}$ is the steady state nucleation rate and $u$ the growth rate. Both $I_{s s}$ and $u$ are inversely proportional to the viscosity, implying $t_{x} \propto \eta$, which was experimentally verified by Mukherjee et al. [26]. The critical cooling rate is inversely proportional to $t_{x}$, indicating $R_{c} \propto 1 / \eta$ [26]. This implies that in order to explain the crystallization in the splat-cooled sample by the strain rate effect on viscosity, the viscosity during splat cooling must be at least 4 orders of magnitude lower than the viscosity during $5 \mathrm{~mm}$ copper mold casting. Strain rate effects on viscosity were quantified for a $\mathrm{Zr}$-based BMG by Lu et al. [27]. It was found that the strain rate effects on viscosity are most pronounced at low temperatures around $T_{g}$ and become less significant as the temperature approaches $T_{x}$. For the highest temperature region studied in his work, which itself is still much lower than the expected crystallization temperature region for $\mathrm{Au}_{49} \mathrm{Ag}_{5.5} \mathrm{Pd}_{2.3} \mathrm{Cu}_{26.9} \mathrm{Si}_{16.3}$ [28], the viscosity dependence on strain rate is $\left|\frac{d \log \eta}{d \log \dot{\varepsilon}}\right|=\frac{1}{4}$. A dependence of $\left|\frac{d \log \eta}{d \log \dot{\varepsilon}}\right|=1$ is required to account for the experimental finding. This suggests that the observed large difference in crystallization kinetics of the differently processed samples cannot be explained by a crystallization rate enhancement due to the previously reported strain rate effect on the viscosity alone. To investigate the origin of strain rate induced crystallization in metallic glasses, one must explore other possible influences on crystallization kinetics.

Phase separation and crystallization driven by large strain rates and related non-Newtonian liquid behavior are common in many complex fluids (see, e.g., [29,30]). The origin for strain rate effects on phase separation and crystallization in such liquids stems from the bonding nature and the size (or length) of the molecules, which is not applicable to metallic liquids. Even though many BMG systems are known to phase separate [31-35], the relation- 
TABLE I. Summary of processing parameters for $\mathrm{Au}_{49} \mathrm{Ag}_{5.5} \mathrm{Pd}_{2.3} \mathrm{Cu}_{26.9} \mathrm{Si}_{16.3}$ alloy and the resulting structures.

\begin{tabular}{lcccc}
\hline \hline \multicolumn{1}{c}{ Sample } & Cooling rate $(\mathrm{K} / \mathrm{s})[19]$ & Processing temperature $(\mathrm{K})$ & Strain rate $\left(\mathrm{s}^{-1}\right)$ & Structure \\
\hline $50 \mu \mathrm{m}$ splat foil & $10^{6}$ & $\sim 900$ & $10^{4}$ & Crystalline \\
$300 \mu \mathrm{m}$ splat foil & $3 \times 10^{4}$ & $\sim 900$ & $4 \times 10^{3}$ & Partially crystalline \\
$50 \mu \mathrm{m}$ splat foil & $10^{6}$ & $\sim 1500$ & $10^{4}$ & Mainly amorphous \\
$30 \mu \mathrm{m}$ cast foil & $10^{6}$ & $\sim 900$ & Negligible & Fully amorphous \\
$5 \mathrm{~mm}$ cast strip & $10^{2}$ & $\sim 900$ & Negligible & Fully amorphous \\
$5 \mathrm{~mm}$ cast strip & $10^{2}$ & $\sim 1500$ & Negligible & Fully amorphous \\
\hline \hline
\end{tabular}

ship between strain rate effects and phase separation in metallic glass liquids has never been established.

The effect of strain rate on metallic liquid includes the effect of elastic energy storage under flow. This is considered in the Gibbs free energy, $G$, as an elastic energy term, $E_{\mathrm{el}}$. The maximum value of this contribution can be estimated, in our case, to $E_{\mathrm{el}}^{\mathrm{max}}=\frac{1}{2} \sigma_{y} \varepsilon_{\mathrm{el}}$, where $\sigma_{y}$ is the room temperature yield strength and $\epsilon_{\mathrm{el}}$ is the elastic strain limit. A liquid subjected to a strain rate can release $E_{\mathrm{el}}$ and thereby lower its free energy by lowering its overall viscosity. BMG-forming alloys are known to have high viscosities [36-38], much higher than the viscosity of each individual elemental constituents. Therefore, it is reasonable to assume that a homogeneous BMG forming liquid could lower its overall viscosity by phase separating into two or more chemically distinct liquids. Such a phase separation process would then release elastic energy, and this elastic energy reduction would act as a driving force for the phase separation process. This approach to explain the influence of strain rate on the crystallization is sketched in Fig. 3. For simplicity, the viscosity of the phaseseparated regions are assumed to be identical. The applied strain rate during solidification phase separates the material of composition $c_{n}$ into $c_{1}$ and $c_{2}$, which is driven by lowering the viscosity from $\eta_{n}$ to $\eta_{\text {dec }}$.

The varying chemical composition of the separated regions directly influences crystallization. In particular, $\mathrm{Au}_{49} \mathrm{Ag}_{5.5} \mathrm{Pd}_{2.3} \mathrm{Cu}_{26.9} \mathrm{Si}_{16.3}$ is known to have a strong dependence of the glass-forming ability on the composition [11]. Our results imply that a phase separation process in this alloy drives the local compositions in the separated region far enough out of the glass-forming composition that even a cooling rate of $10^{6} \mathrm{~K} / \mathrm{s}$ is insufficient to avoid crystallization. This scenario is represented by the change in critical cooling rate, $R_{c}$, which is the right axis of Fig. 3. The strain rate during splat cooling of $10^{4} \mathrm{~s}^{-1}$ is capable of phase separating the initial composition, $c_{n}$ into $c_{1}$ and $c_{2}$, both with larger critical cooling rates. If now the cooling rate is below $R_{c}^{\text {dec }}$, the material phase separates and crystallizes during solidification. It has been predicted [22] and observed in several systems that crystallization takes place in shear bands where plastic shear is localized [39,40], resulting in a very high strain rate. If the applied cooling rate remains larger than effective $R_{c}^{\text {dec }}$, crystallization during solidification can be avoided and the material would only phase separate during solidification. The finding by Cao et al. [41] that phase separation takes place in shear bands with no evidence of crystallization might be an example of this scenario.

The extent to which the strain rate applied during solidification of the BMG influences crystallization depends on the relative influence of the elastic energy term in comparison to the enthalpic and entropic terms in $G$. Additionally, the extent to which the composition change during phase separation affects the glass-forming ability depends on the composition dependence of the GFA. For example, the well-studied Zr-based and Pd-based BMGs can be expected to show a less pronounced strain rate effect on the crystallization since they possess a wide glass-forming region on the compositional space $[42,43]$. In addition, the directions in the compositional space for both (i) phase separation process driven by the lowering of elastic energy and (ii) the composition sensitivity of the GFA must coincide in order to result in a strong influence of the strain rate on the crystallization kinetics. The directional concurrence between (i) and (ii) controls the degree of the strain rate effect on crystallization.

It should be mentioned that the phase separation process described in the present approach is different from the previously reported decomposition processes in metallic

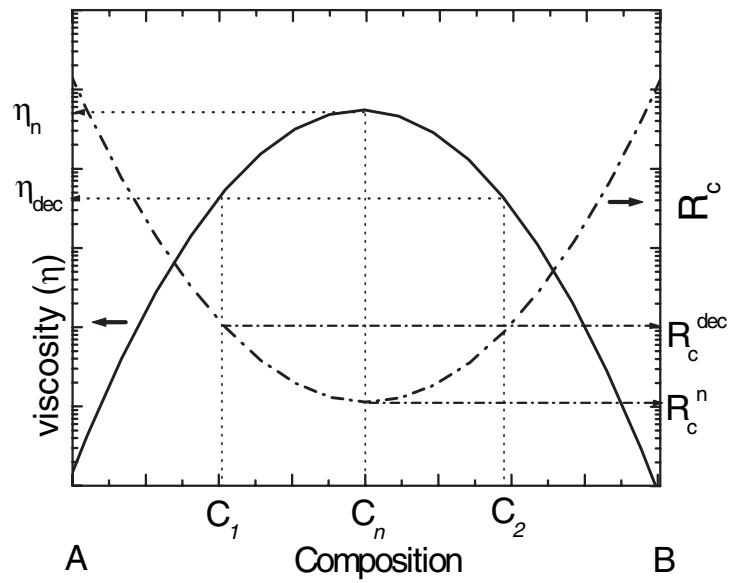

FIG. 3. Sketch of the approach to explain the influence of strain rate on the crystallization. The applied strain rate during solidification causes the material of composition $c_{n}$ to phase separate into $c_{1}$ and $c_{2}$ by lowering the viscosity from $\eta_{n}$ to $\eta_{\mathrm{dec}}$. If the applied cooling rate is below $R_{c}^{\mathrm{dec}}$, the material separates and crystallizes during solidification. If the applied rate is above $R_{c}^{\text {dec }}$, crystallization could be avoided during solidification and the material only phase separates. 
glasses [31-35]. In those processes, the driving force for chemical decomposition comes from a lowering of the enthalpic contribution of $G$. The maximum driving force for a strain rate driven decomposition process can be estimated for a Pt-based BMG to be $E_{\mathrm{el}}^{\max } \approx 100 \mathrm{~J} / \mathrm{mol}$, using $\sigma_{y}=1400 \mathrm{MPa}$ and $\epsilon_{\mathrm{el}}=0.014$ [44]. This number is comparable to the driving force for the previously reported chemically driven decomposition process of approximately $100 \mathrm{~J} / \mathrm{mol}$ [45].

In summary, this Letter reports on the experimental evidence of a strain rate induced crystallization in a metallic system during cooling from the melt. Previously reported strain rate effects on viscosity alone are not sufficient to explain this finding. A qualitative approach is suggested where the strain rate induces phase separation in the homogeneous liquid. If phase-separated chemical compositions are driven out of the glass-forming region for the applied cooling rate, the material crystallizes during cooling. In the present case, a cooling rate as high as $10^{6} \mathrm{~K} / \mathrm{s}$ is not sufficient to suppress crystallization. This work suggests that a strain-rate-dependent critical cooling rate must be considered in order to assess the intrinsic glass-forming ability of metallic liquids.

*Corresponding author.

Electronic address: schroers@caltech.edu

[1] D. Turnbull and R. E. Cech, J. Appl. Phys. 21, 804 (1950).

[2] W. Klement, R.H. Willens, and P. Duwez, Nature (London) 187, 869 (1960).

[3] H. H. Liebermann, Mater. Sci. Eng. 43, 203 (1980).

[4] S. A. Miller and R. J. Murphy, Scr. Metall. 13, 673 (1979).

[5] H. S. Chen, Acta Metall. 22, 1505 (1974).

[6] A. Inoue, T. Zhang, and T. Masumoto, Mater. Trans., JIM 30, 965 (1989).

[7] A. Peker and W. L. Johnson, Appl. Phys. Lett. 63, 2342 (1993).

[8] V. Ponnambalam, S. J. Poon, G. J. Shiflet, V. M. Keppens, R. Taylor, and G. Petculescu, Appl. Phys. Lett. 83, 1131 (2003).

[9] J. Schroers and W. L. Johnson, Appl. Phys. Lett. 84, 3666 (2004).

[10] D. H. Xu, B. Lohwongwatana, G. Duan, W. L. Johnson, and C. Garland, Acta Mater. 52, 2621 (2004).

[11] J. Schroers, B. Lohwongwatana, W. L. Johnson, and A. Peker, Appl. Phys. Lett. 87, 061912 (2005).

[12] J. Schroers and W. L. Johnson, Appl. Phys. Lett. 80, 2069 (2002).

[13] A. Inoue, N. Nishiyama, and H. Kimura, Mater. Trans., JIM 38, 179 (1997).

[14] M. C. Lee, J. M. Kendall, and W. L. Johnson, Appl. Phys. Lett. 40, 382 (1982).

[15] X. L. Yeh, K. Samwer, and W. L. Johnson, Appl. Phys. Lett. 42, 242 (1983).

[16] J. H. Perepezko and J. S. Smith, J. Non-Cryst. Solids 44, 65 (1981).
[17] A. Blatter and M. von Allmen, Phys. Rev. Lett. 54, 2103 (1985).

[18] A. Inoue, Acta Mater. 48, 279 (2000).

[19] The cooling rate is estimated from solution to the heat flow equation for a plate of liquid alloy cooled by heat conduction to a thick mold which yields $R_{c}^{\text {plate }}=$ $0.4 K_{t} T_{l} / c_{p} L^{2}$, with $K_{t}$ as the thermal conductivity, $c_{p}$ the specific heat of the alloy, and $L$ the plate's thickness.

[20] Calculated from the time required to deform a liquid drop of $1 \mathrm{~mm}$ diameter sphere to a splat $50 \mu \mathrm{m}$ thick. The time is of the order of ms.

[21] F. Spaepen, Acta Metall. 25, 407 (1977).

[22] A. S. Argon, Acta Metall. 27, 47 (1979).

[23] H. Kato, Y. Kawamura, A. Inoue, and H. S. Chen, Appl. Phys. Lett. 73, 3665 (1998).

[24] T. G. Nieh, C. A. Schuh, J. Wadsworth, and Y. Li, Intermetallics 10, 1177 (2002).

[25] D. R. Uhlmann, J. Non-Cryst. Solids 7, 337 (1972).

[26] S. Mukherjee, J. Schroers, W. L. Johnson, and W.-K. Rhim, Phys. Rev. Lett. 94, 245501 (2005).

[27] J. Lu, G. Ravichandran, and W. L. Johnson, Acta Mater. 51, 3429 (2003).

[28] During cooling, BMGs crystallize at or above the nose in the time temperature transformation diagram; see e.g., J. Schroers, A. Masuhr, W.L. Johnson, and R. Busch, Phys. Rev. B 60, 11855 (1999).

[29] J.H. Han and C. D. Han, Polym. Eng. Sci. 28, 1616 (1988).

[30] C. R. Safinya, E. B. Sirota, and R. J. Plano, Phys. Rev. Lett. 66, 1986 (1991).

[31] R. Busch, S. Schneider, A. Peker, and W. L. Johnson, Appl. Phys. Lett. 67, 1544 (1995).

[32] M.P. Macht, N. Wanderka, A. Wiedenmann, H. Wollenberger, Q. Wei, H. J. Fecht, and S. G. Klose, Mater. Sci. Forum 225, 65 (1996).

[33] J.F. Loffler and W. L. Johnson, Mater. Sci. Eng. A 304, 670 (2001).

[34] J. F. Miller, T. D. Shen, and R. B. Schwarz, Intermetallics 10, 1047 (2002).

[35] A. A. Kündig, M. Ohnuma, D. H. Ping, T. Ohkubo, and K. Hono, Acta Mater. 52, 2441 (2004).

[36] L. Shadowspeaker and R. Busch, Appl. Phys. Lett. 85, 2508 (2004).

[37] S. Mukherjee, J. Schroers, Z. Zhou, W. L. Johnson, and W.-K. Rhim, Acta Mater. 52, 3689 (2004).

[38] H. Tanaka, J. Non-Cryst. Solids 351, 678 (2005).

[39] H. Chen, Y. He, G. J. Shiflet, and S. J. Poon, Nature (London) 367, 541 (1994).

[40] J. J. Kim, Y. Choi, S. Suresh, and A.S. Argon, Science 295, 654 (2002).

[41] Q. Cao, J. Li, Y. Zhou, and J. Jiang, Appl. Phys. Lett. 86, 081913 (2005).

[42] T. D. Shen, Y. He, and R. B. Schwarz, J. Mater. Res. 14, 2107 (1999).

[43] T. A. Waniuk, J. Schroers, and W. L. Johnson, Appl. Phys. Lett. 78, 1213 (2001).

[44] J. Schroers and W. L. Johnson, Phys. Rev. Lett. 93, 255506 (2004).

[45] Y.J. Kim, R. Busch, W. L. Johnson, A. J. Rulison, and W. K. Rhim, Appl. Phys. Lett. 68, 1057 (1996). 